\title{
A Rare Cavernous Metastasis of a Bladder Tumor
}

\author{
Gregoire Assenmacher ${ }^{1 *}$, Fouad Aoun ${ }^{1}$, Elea Deplaen ${ }^{1}$, Alexandre Peltier $^{1}$, Amine Slaoui $^{2}$, Nicolas Sirtaine ${ }^{3}$ and Thierry \\ Roumeguere $^{4}$ \\ ${ }^{1}$ Department of Urology, Institut Jules Bordet, Free University of Brussels, Belgium \\ ${ }^{2}$ Department of Urology, Ibn Sina Hospital, Mohamed V University, Morocco \\ ${ }^{3}$ Department of Pathology, Institut Jules Bordet, Free University of Brussels, Belgium \\ ${ }^{4}$ Urology Department, Erasmus Hospital, Free University of Brussels, Belgium
}
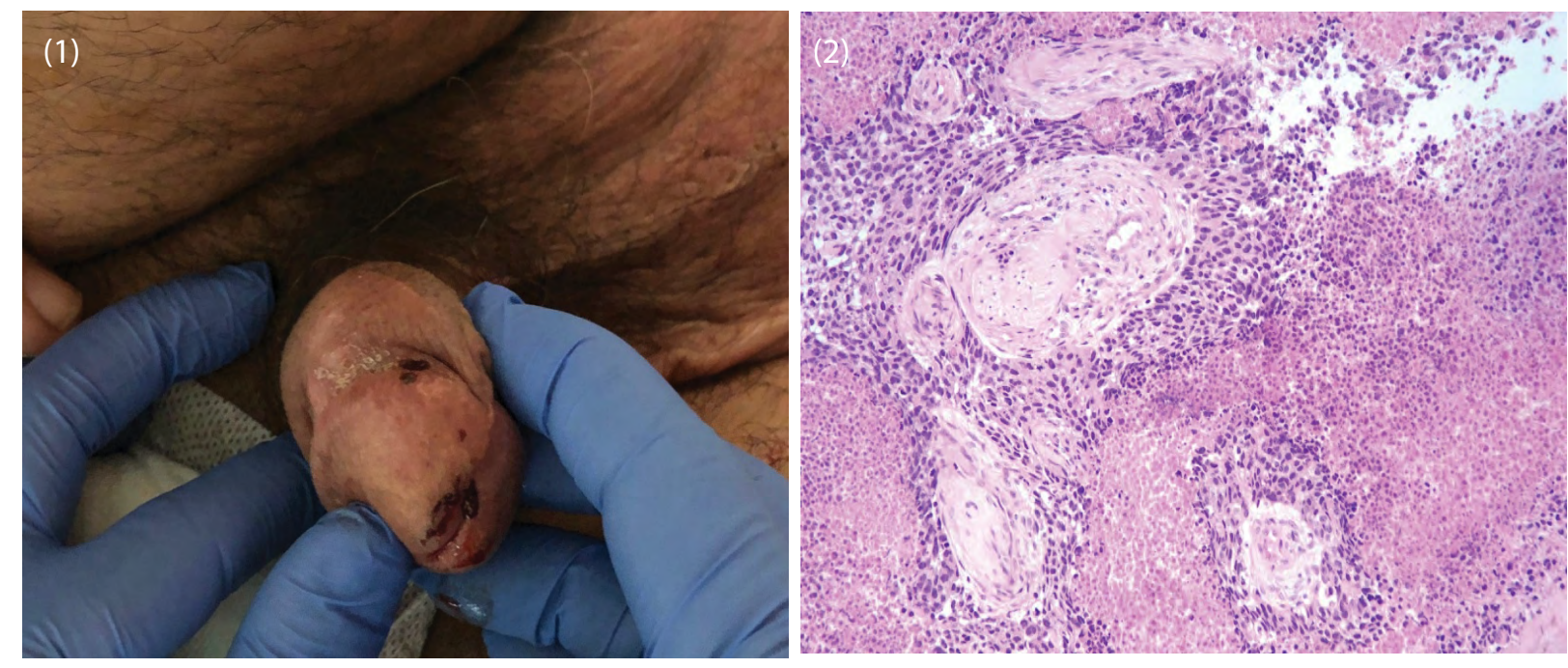

Figure 1: Latero-penial induration with tumor material outlet by the ureter.

Figure 2: (H\&E X200) Largely necrotic undifferentiated carcinomatous proliferation. Note perinervous infiltration pattern.

\section{Clinical Image}

Surgically treated bladder tumors have a poor prognosis. Indeed, it is $50 \%$ of survival at 5 years for $\mathrm{T} 2$ tumors. The literature reports some rare cases of metastasis after radical treatment. The cavernous localization remains extremely rare (approximately 1\%). It can occur from 8 months to 10 years after surgery. Here, we report the case of a 74-year-old chronic smoking patient who, in August 2015, underwent a total cystoprostatectomy. Two years later he presents an induration of the penis (Figure 1), with no other associated sign. On clinical examination we find inflammatory signs with a beginning of infection. Given the clinical context, a biopsy was performed and came back in favor of a cavernous metastasis of urothelial carcinomas (Figure 2). Chemotherapy and immunotherapy based on Cisplatin - Gemcitabine and Atezolizumab were started but the patient died a few weeks later.

*Corresponding author: Gregoire Assenmacher, Department of Urology, Institut Jules Bordet, Free University of Brussels, Belgium, Tel: 0032474243910 , E-mail: greg.assenmacher@gmail.com

Citation: Assenmacher G, Aoun F, Deplaen E, Peltier A, Slaoui A, et al. (2018) A Rare Cavernous Metastasis of a Bladder Tumor. Int J Clin Med Imaging 4: 599. doi:10.4172/2376-0249.1000599

Copyright: (C) 2018 Assenmacher G, et al. This is an open-access article distributed under the terms of the Creative Commons Attribution License, which permits unrestricted use, distribution, and reproduction in any medium, provided the original author and source are credited. 